Western University

Scholarship@Western

2019

\title{
Thermo-responsive and covalently cross-linkable hydrogels for intra-articular drug delivery
}

\author{
Andy Prince \\ The University of Western Ontario \\ Ian J. Villamagna \\ Western University \\ Aneta Borecki \\ Western University \\ Frank Beier \\ Western University \\ John R. de Bruyn \\ Western University
}

See next page for additional authors

Follow this and additional works at: https://ir.lib.uwo.ca/chempub

Part of the Chemistry Commons

Citation of this paper:

Prince, Andy; Villamagna, Ian J.; Borecki, Aneta; Beier, Frank; de Bruyn, John R.; Hurtig, Mark; and Gillies, Elizabeth, "Thermo-responsive and covalently cross-linkable hydrogels for intra-articular drug delivery" (2019). Chemistry Publications. 128.

https://ir.lib.uwo.ca/chempub/128 


\section{Authors}

Andy Prince, Ian J. Villamagna, Aneta Borecki, Frank Beier, John R. de Bruyn, Mark Hurtig, and Elizabeth Gillies 


\section{Correction to "Thermo-responsive and covalently cross-linkable hydrogels for intra-articular drug delivery"}

David Andrew Prince, ${ }^{a}$ Ian J. Villamagna, ${ }^{\text {,c }}$ Aneta Borecki, ${ }^{\text {a,d }}$ Frank Beier,, ,e John R. de Bruyn, d,f Mark Hurtig,, and Elizabeth R. Gillies*a,b,c,d,h

aDepartment of Chemistry, The University of Western Ontario, 1151 Richmond St., London, Ontario, Canada, N6A 5B7

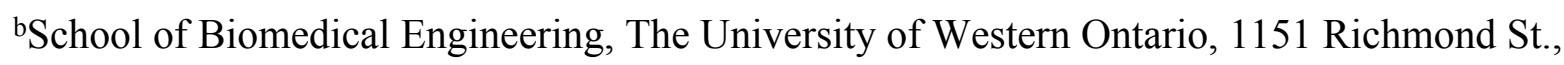
London, Ontario, Canada, N6A 5B9

${ }^{\mathrm{c} B o n e}$ and Joint Institute, The University of Western Ontario

${ }^{\mathrm{d} C e n t r e}$ for Advanced Materials and Biomaterials Research, The University of Western Ontario

eDepartment of Physiology and Pharmacology, The University of Western Ontario, 1151

Richmond St., London, Ontario, Canada, N6A 3B7

fDepartment of Physics and Astronomy, The University of Western Ontario, 1151 Richmond St.

London, Ontario, Canada, N6A 3K7

gOntario Veterinary College, Department of Clinical Studies, University of Guelph, 50 Stone

Road, Guelph, Ontario, Canada N1G 2W1

hDepartment of Chemical and Biochemical Engineering, The University of Western Ontario, 1151 Richmond St., London, Ontario, Canada, N6A 5B9

*Author to whom correspondence should be addressed: E-mail: egillie@uwo.ca 
ACS Appl. Bio Mater. 2019, 2 (8), 3498-3507. DOI: 10.1021/acsabm.9b00410

In the course of our ongoing research, we discovered that an error was made in the calculation of the compressive moduli of the hydrogels, which resulted in values 10 -fold lower that the actual values. The corrected values are now provided in updated versions of Table 2 and Figure 5. The supporting information has also been updated to reflect the corrected moduli (Table S2 and Figure S19). These changes do not significantly alter the scientific conclusions of the paper. We apologize for the error.

Table 2. G', G", and compressive moduli of drug-free and CXB-loaded (5 wt $\%$ ) $\mathbf{1 . 5} \mathbf{k}_{\mathbf{3 1 5 0}}$-MA hydrogels, measured after $60 \min$ at $37^{\circ} \mathrm{C}$.

\begin{tabular}{|c|c|c|c|}
\hline & G' $^{\prime}(\mathrm{kPa})$ & G" (kPa) & $\begin{array}{l}\text { Compressive } \\
\text { modulus (kPa) }\end{array}$ \\
\hline $1.5 \mathrm{k}_{3150}-\mathrm{MA}$ & $7.7 \pm 1.2$ & $1.2 \pm 0.7$ & $190 \pm 10$ \\
\hline $1.5 \mathrm{k}_{3150}-\mathrm{MA}+5 \% \mathrm{CXB}$ & $17 \pm 8$ & $2.8 \pm 1.5$ & $190 \pm 10$ \\
\hline
\end{tabular}




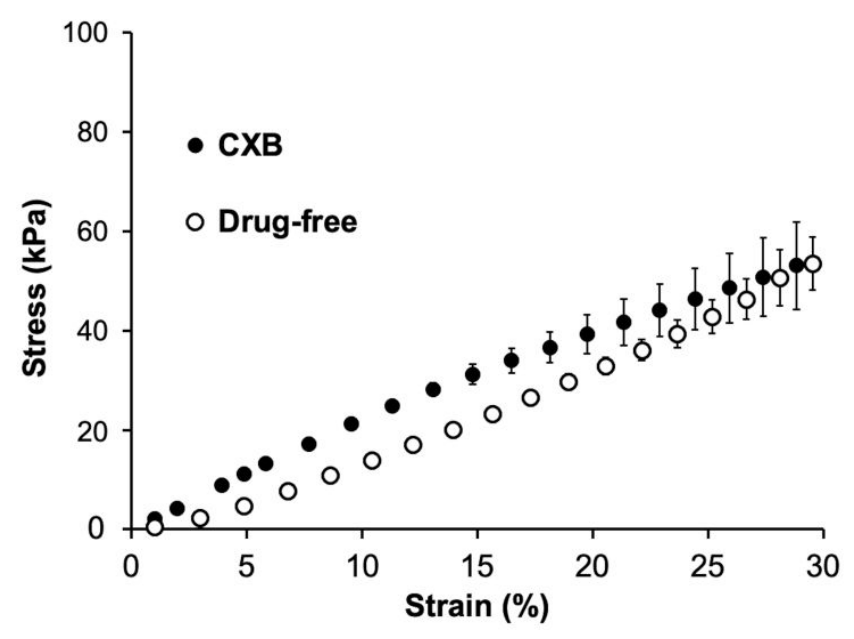

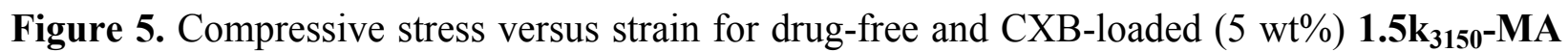
hydrogels. Error bars correspond to standard deviations $(\mathrm{N}=3)$. 